\title{
Les écoles-acteurs en Guadeloupe, un espace de débat sur les pollutions agricoles et le devenir de l'agriculture
}

\author{
Jean-Philippe Tonneau ${ }^{1,5,{ }^{*}}$, Vincent Bonnal ${ }^{1,2,5}$, Jeremy Bourgoin ${ }^{1,5}$, Agathe Cheval ${ }^{2,5}$, \\ Magalie Jannoyer ${ }^{3}$, Jean-Pierre Chéry ${ }^{4,5}$ et Philippe Cattan ${ }^{1,5}$ \\ ${ }^{1}$ CIRAD, UMR TETIS, F-34398 Montpellier, France \\ 2 CIRAD, UMR TETIS, F-97130 Capesterre-Belle-Eau, Guadeloupe, France \\ ${ }^{3}$ CIRAD, DGDRS, Avenue Agropolis, F-34398 Montpellier, France \\ ${ }^{4}$ AgroParisTech, UMR TETIS, Montpellier, France \\ 5 TETIS, Univ Montpellier, AgroParisTech, CIRAD, CNRS, IRSTEA, Montpellier, France
}

\begin{abstract}
Résumé - Le projet RIVAGE veut favoriser l'adoption de pratiques alternatives pour gérer les impacts de la pollution diffuse dans le bassin versant de la rivière Pérou en Guadeloupe. Son objectif est de produire et partager les connaissances sur les processus, les impacts et les pratiques innovantes avec les acteurs du territoire. Pour faciliter la prise en compte des résultats, le projet a créé une « école-acteurs ». L'école-acteurs est un espace d'échanges autour des thématiques liées à la pollution diffuse agricole. Le concept d'écoleacteurs fait référence aux théories d'éducation populaire et d'apprentissage collectif. L'article rappelle ces références théoriques et les utilise dans un processus d'auto-évaluation des premiers résultats obtenus par l'école-acteurs. Les auteurs dressent quelques enseignements et orientations pour le futur.
\end{abstract}

Mots clés : Guadeloupe / pollution agricole / politiques publiques / forum / apprentissage

\begin{abstract}
Learning partners schools in Guadeloupe: A tool for debate on agricultural pollution and the future of agriculture. The RIVAGE project aims to promote the adoption of alternative practices to manage the impacts of diffuse pollution in the Pérou River watershed in Guadeloupe. The objective is to produce and share knowledge on processes, impacts and innovative practices with the actors of the territory. To facilitate results uptake, the project has created a "learning partners school". The learning partners school is a space for exchanges, a forum, developed around themes related to agricultural diffuse pollution. The concept of learning partners school is built on the theories of popular education and collective learning. The article recalls these theoretical references and uses them in a self-evaluation process of the first results obtained by the learning partners school. The authors draw up lessons and orientations for the future.
\end{abstract}

Keywords: Guadeloupe / agricultural pollution / public policies / forum / learning

\section{Pollution diffuse en Guadeloupe : le besoin de politiques innovantes}

En Guadeloupe, les filières d'exportation, canne à sucre et banane, avec respectivement 13400 et 1800 ha en 2018 (DAAF, 2018a, 2018b), côtoient une agriculture dite de « diversification » d'environ 15000 ha. Les systèmes intensifs canne et banane sont souvent associés à des impacts négatifs sur l'environnement. Le plus emblématique est la pollution à la chlordécone, un insecticide organochloré épandu en bananeraie et interdit d'usage depuis 1993 (Lesueur-Jannoyer et al.,

\footnotetext{
*Auteur de correspondance $:$ tonneaujp@wanadoo.fr
}

2016), pour laquelle il n'existe aucun procédé de réduction. Elle contamine durablement les milieux et les chaînes trophiques (sols, eaux, végétaux et animaux) (LesueurJannoyer et al., 2012). Les effets sur la santé humaine sont avérés: augmentation du risque de cancer de la prostate, perturbation du développement chez les jeunes enfants... (Multigner et al., 2016). Les mesures de gestion des impacts prises par l'État (restriction de commercialisation des produits agricoles et de la mer; usages restrictifs des eaux et des sols) ont suscité de fortes oppositions et des conflits entre acteurs.

Pour diminuer le recours aux pesticides, la gestion mécanique des adventices et les plantes de couverture sont proposées (Meiffren et al., 2014). Mais l'adoption par les agriculteurs est inégale (Della Rossa et al., 2020) et pose la 
question du changement de pratiques aux échelles à la fois individuelle et du territoire. C'est au niveau des bassins versants que les dispositifs d'accompagnement des producteurs doivent se mettre en place (Barbier et Larrue, 2011) et c'est à l'échelle de l'île que doivent être conçues les politiques publiques.

Les instances de concertation existantes se sont essentiellement développées dans le cadre des filières qui, aux Antilles, traitent les questions de pollution diffuse (Della Rossa et al., 2020). Les filières concentrent d'ailleurs l'essentiel des appuis aux agriculteurs (Deffontaines et al., 2020). Dans un souci légitime de maintien des niveaux de production, l'information diffusée, essentiellement technique et spécifique à la filière, privilégie l'utilisation rationnelle des pesticides.

Le projet RIVAGE (Innover en agroécologie pour gérer, préserver et restaurer la qualité environnementale du territoire) a accompagné les agriculteurs dans l'adoption de pratiques agroécologiques. Très vite, la mise en œuvre de son volet "Communiquer et diffuser les informations liées aux pollutions agricoles " s'est heurtée à de fortes divergences sur les actions à mener et sur la nécessité d'organisations territoriales, ressenties comme une remise en cause des organisations filières.

Forte de ce constat, l'équipe du projet a souhaité favoriser un débat transversal aux filières, premier pas avant la définition de plans d'action articulant toutes les initiatives sur les bassins versants. C'est l'objet des «écoles-acteurs » (EA).

L'article analyse en quoi les écoles-acteurs modifient la nature des concertations autour de la pollution agricole diffuse et favorisent de nouvelles modalités de partage de l'information. Nous présentons, en première partie, le dispositif en nous référant aux théories d'éducation populaire et d'apprentissage collectif. Dans une deuxième partie, nous précisons la nature et les modes de fonctionnement de l'EA. Nous tirons quelques enseignements et des propositions dans une troisième partie.

\section{La construction des politiques publiques en situation de crise}

Dans une situation de crise comme celle des pollutions diffuses en Guadeloupe, l'élaboration de politiques publiques est difficile. Les marges de manœuvre sont faibles : les terres agricoles sont rares (32\% de SAU en Guadeloupe), les intérêts sont divers et les lobbies sont puissants (Bonin et Cathelin, 2014). La crise révèle les fragilités démocratiques de la prise de décision (Revault d'Allonnes, 2011). Le manque de connaissances sur les phénomènes et sur leurs impacts, toujours complexes, fait que la décision indiscutable n'existe pas et que sa légitimité est toujours remise en cause. De fait, la crise renforce la distanciation entre pouvoirs et populations.

\subsection{Territorialisation et participation}

Pour atténuer cette distanciation, depuis trois décennies, la construction des politiques publiques a favorisé la territorialisation et la participation (Barbier et Larrue, 2011; Salles et al., 2006).

La directive-cadre européenne sur l'eau a fait émerger le concept d' «hydro-territoires » (Barbier et Roussary, 2016) : d'une part le bassin versant, pertinent pour évaluer les impacts des pratiques sur le milieu et notamment sur les eaux souterraines et de surface, et d'autre part l'hydro-territoire de gestion (agence de bassin, département, région...), où les coordinations et les moyens se mettent en place.

Parallèlement, l'État a développé une gestion plus intégrée et participative (Salles et al., 2006), basée sur la concertation et la négociation entre différentes catégories d'acteurs, porteurs d'enjeux de natures différentes (Mongruel et al., 2011). Les acteurs se retrouvent alors dans des instances de concertation ou des forums qui alimentent la réflexion politique en proposant des modalités d'action (Fouilleux, 2000).

\subsection{Les limites des processus de concertation}

L'une des critiques les plus couramment adressées aux processus de concertation est qu'ils prennent peu en compte la complexité des contextes socio-politiques (Cooke et Kothari, 2001; Mathevet et al., 2010). Ces contextes sont caractérisés par l'existence de conflits d'intérêt et de rapports de force souvent inégaux (Barnaud et al., 2016). Certains acteurs peuvent contrôler les dispositifs participatifs. Ils disposent du pouvoir d'inclure ou d'exclure d'autres acteurs dans la négociation, de choisir des enjeux et des thèmes, d'imposer leurs idées dans les discussions en ignorant ou en dominant les opinions d'autres protagonistes, de mettre en œuvre des décisions sans respecter les accords trouvés pendant la négociation (Faysse, 2006).

\subsection{Une concertation accompagnée}

Face à cette constatation, Barnaud et al. (2016) distingue deux grandes stratégies, les approches critiques et les approches dialogiques.

Les approches critiques (Ulrich, 2003) préconisent de gérer les inégalités préexistantes pour éviter que les processus participatifs ne creusent les inégalités. Une démarche participative ne peut rester neutre et doit permettre à tous les acteurs de s'exprimer et d'être entendus dans les débats. Cela passe principalement par un renforcement spécifique des compétences des acteurs absents ou faiblement présents dans le débat.

Pour les tenants de l'approche dialogique (Roling et Wagemakers, 1998), le principal obstacle est le manque de compréhension mutuelle. Une fois levées les barrières de communication, les protagonistes peuvent avoir une représentation partagée de la situation et arriver à un consensus, nécessaire pour une action collective durable.

Toujours selon ce courant dialogique (Muller, 2000), les médiateurs facilitent les interactions entre les acteurs en créant des espaces de dialogue qui favorisent la co-production de connaissances, la compréhension et la reconnaissance de positions différentes, parfois fortement contrastées (Leclerc, 2000). Un médiateur accompagne les acteurs sans jamais proposer de solutions ni prendre de positions hégémoniques, en évitant des postures de courtier du développement (Olivier de Sardan et Bierschenk, 1994).

Dans cette perspective, le processus est essentiel. Pour Jobert (1994), l'accord sur une méthode de travail suffit pour initier un travail en commun, sans nécessairement être d'accord sur les objectifs. Débattre des méthodes de travail, 
d'une feuille de route, permet d'aboutir à un premier accord donnant confiance tout en laissant de côté les éléments les plus conflictuels (Tonneau et al., 2011).

C'est en fonction de ces réflexions théoriques que nous avons construit les écoles-acteurs.

\section{L'école-acteurs}

\subsection{Genèse des écoles-acteurs}

Le terme « école-acteurs » est nôtre. Le nom est dérivé des écoles-chercheurs (Werdenberg et Arrault, 2014). Comme elles, les écoles-acteurs privilégient les interactions entre les participants en alternant des séquences d'apports conceptuels, des témoignages, des débats et des ateliers de travail. Une école-acteurs combine des apports de connaissances et des dynamiques de groupe, notamment interdisciplinaires. Elle met en lien des acteurs en leur donnant l'occasion de se rencontrer ailleurs et de travailler de manière différente.

L'école-acteurs fait aussi référence à l'éducation populaire définie comme «ensemble des démarches d'apprentissage et de réflexion critique par lesquelles des citoyens et citoyennes mènent collectivement des actions qui amènent une prise de conscience individuelle et collective» (Maurel, 2001). Nos écoles-acteurs se basent sur la mutualisation, la valorisation et la mise en débat d'expériences et de réflexions plurielles, principes clefs de l'éducation populaire (Gorry, 2012).

Ces références se sont traduites dans les hypothèses de travail suivantes :

- Des médiateurs peuvent favoriser les alliances nécessaires à la définition de mesures et de politiques publiques autour de la pollution agricole diffuse.

- Un processus collectif de réflexion et d'apprentissage permet aux acteurs de partager les connaissances tout en prenant conscience de la complexité des situations.

- Ce processus d'apprentissage s'appuie sur une représentation systémique et partagée de la pollution diffuse.

- Ce processus est alimenté par la mise à disposition de connaissances accessibles et d'expériences référencées.

\subsection{L'animation des écoles-acteurs}

L'équipe d'animation était constituée de 5 chercheurs du CIRAD et d'AgroParisTech.

La difficulté du projet RIVAGE à diffuser ses résultats a conduit ces chercheurs à proposer un espace d'échange et une méthode, acceptés par les partenaires. Jamais le rôle de l'équipe d'animation n'a été contesté. Les participants ont implicitement reconnu que les chercheurs étaient des médiateurs légitimes. Leur parti pris de neutralité était crédible du fait soit de leurs travaux antérieurs sur la pollution soit de la distanciation géographique qui les éloignait des rapports de force locaux. Deux des membres de l'équipe d'animation avaient une très bonne connaissance des mécanismes de pollution diffuse et du paysage institutionnel en Guadeloupe. Les plus impliqués dans la conduite des sessions maîtrisaient les techniques d'animation, compte tenu de leurs expériences passées.

L'équipe d'animation a pris la responsabilité de l'organisation des EA en lançant les invitations, en proposant les thèmes, en choisissant les intervenants, en facilitant la participation des agriculteurs et de la société civile, en sélectionnant des expériences alternatives, en animant les débats, en rédigeant les synthèses. Cela s'est traduit concrètement par le séquençage de l'atelier.

Les intervenants lors de l'introduction et de la contextualisation ont principalement utilisé un mode expositif (par ex. présentations), parfois interactif (par ex. forums de posters). Mais une fois ce cadre défini, l'école-acteurs a été un espace de discussion ouvert, principalement dans les débats en groupes ou en plénière. L'équipe d'animation s'est alors intégrée dans le groupe comme participant apprenant (Euvé, 2018), en facilitant le dialogue «science et/en société». (Gorry, 2012).

\subsection{Les participants aux écoles-acteurs}

L'équipe d'animation a d'abord invité les partenaires du projet RIVAGE, qui eux-mêmes ont invité d'autres personnes : élus, techniciens ou conseillers agricoles, représentants des différentes collectivités territoriales et institutions de l'État, des ONG, de la profession. Ce sont eux qui participent habituellement aux arènes technico-scientifiques, mais l'EA les mettait en situation d'apprentissage par le débat.

Deux orientations contribuaient à cet objectif. D'abord, les participants étaient invités «intuitu personae». La grande majorité n'avaient pas de fonction dirigeante. Ils ne représentaient pas leurs institutions et ils n'étaient pas tenus de défendre les positions de ces dernières. La confidentialité des propos était garantie. Les verbatim étaient anonymisés. Ensuite, l'école-acteurs n'avait pas de décisions à prendre ni de plans à élaborer. Ces orientations devaient favoriser le débat et une production de connaissances autant que possible libérés des intérêts spécifiques et des pesanteurs administratives et institutionnelles.

Les EA ont été ouvertes à tous ceux désirant y participer, sans aucune restriction. Les participants d'une session étaient systématiquement invités aux suivantes. Le nombre d'invitations a ainsi crû au fur et à mesure, de 35 en novembre 2016 à 138 en septembre 2019. En moyenne, un tiers des invités ont été présents. L'équipe d'animation a relancé les acteurs jugés compétents pour le sujet débattu (par exemple sollicitation d'un transformateur de produits agricoles pour la session consacrée à l'alimentation). 205 personnes ont participé aux 7 écoles-acteurs, la plupart plusieurs fois. 40 étaient issus de structures d'appui ; 87 étaient des chercheurs ; 36 étaient issus des institutions de l'État; 26 étaient des producteurs et 16 provenaient de la société civile. La participation a été variable, de 13 à 43 personnes. La figure 1 identifie quelques corrélations entre thèmes et nombre/type de participants. L'assistance a été plus nombreuse et plus diversifiée quand les thèmes étaient moins techniques ou spécifiques. Les élus ne se sont jamais déplacés. La participation des agriculteurs et de leurs représentants (17\% sur l'ensemble des sessions) a été plus importante quand ils ont parlé de leurs pratiques. La société civile, absente dans les premières sessions, a été présente lors des dernières sessions.

\subsection{Une école-acteurs : un itinéraire type}

La durée d'une session est d'une grosse demi-journée. Elle est organisée en séquences d'introduction, de contextualisa- 


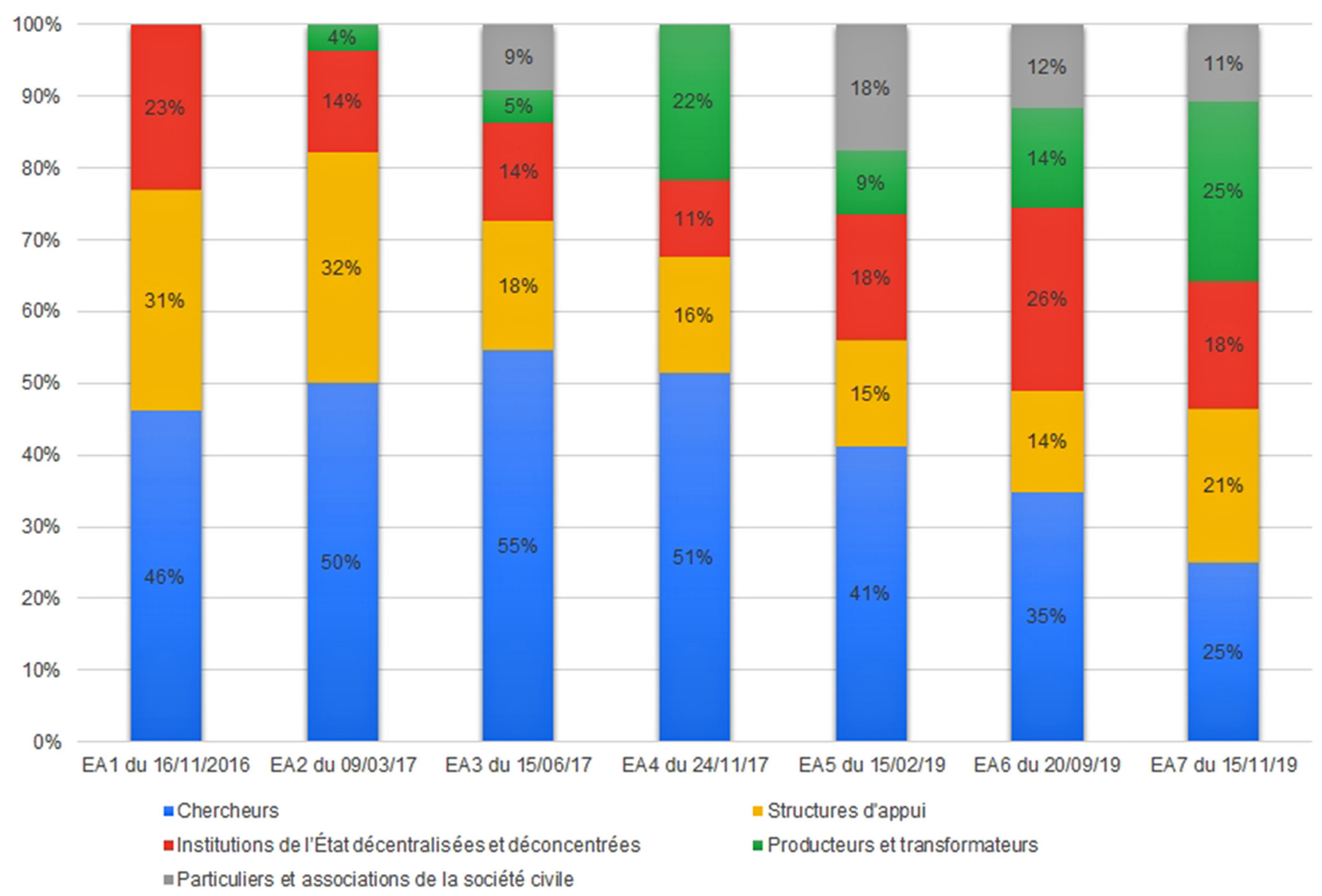

Fig. 1. Évolution de la participation des différents types d'acteurs.

Fig. 1. Participation trends for different types of actors.

tion, de discussion en plénière et d'approfondissement en groupes de travail. Une synthèse présentée par un membre de l'équipe d'animation résume les travaux. Une évaluation orale, à chaud, recueille les ressentis des participants. Un buffet prolonge le débat, et ainsi crée ou entretient des liens entre participants.

Deux principes directeurs guident la séquence de travail : la mise en commun des connaissances et des savoirs; l'écoute bienveillante de tous par tous. La parole se veut libre et toutes les idées méritent attention et discussion.

Les membres de l'équipe d'animation rédigent un verbatim, en anonymisant les prises de parole.

\subsection{Les sessions de l'école-acteurs en Guadeloupe}

Une première série de quatre écoles-acteurs a été organisée entre novembre 2016 et décembre 2017. Après un an d'interruption due à des problèmes de financement, 3 sessions ont ensuite été organisées en 2019. La crise sanitaire a interrompu les travaux en 2020.

Les caractéristiques des sept sessions sont présentées dans le tableau 1: date, nombre et profils des participants, thème, points abordés en détaillant les questionnements, les besoins identifiés et des difficultés rencontrées. Les échanges et débats abordés lors de ces sessions ont permis de récolter un matériel riche et de dégager des propositions d'actions.

\subsection{Le matériel}

Le matériel des verbatim est composé des présentations introductives, des témoignages d'acteurs présentant leurs expériences et leurs pratiques en s'appuyant sur des posters ou non. Des supports/objets intermédiaires ont facilité les interactions entre participants. Ainsi, des cartographies participatives ont identifié les espaces pollués. Des indicateurs ont été élaborés de manière participative, par " pose de post-it » sur des grilles d'analyses matricielles pour identifier les enjeux de l'agriculture en Guadeloupe. Les participants ont aussi exprimé des positions, parfois tranchées, et des propositions. Ces positions et ces propositions étaient résumées par un animateur en fin de chaque session. Cette synthèse reconnaissait et formalisait les désaccords.

\subsection{Bilan des propositions issues des écoles-acteurs}

Les propositions vont bien au-delà de techniques moins polluantes ou de l'interdiction administrative de productions agricoles. Les premières écoles-acteurs étaient pourtant consacrées à ces thèmes (Innovations et dispositifs d'innovation; Comment se passer $d u$ glyphosate?). Mais très rapidement, la question de la communication a été posée par les agriculteurs et les filières («sensibiliser sans culpabiliser »). L'importance d'informer a été réaffirmée en 
Tableau 1. Caractéristiques des écoles-acteurs.

Table 1. Characteristics of learning partners schools.

\begin{tabular}{|c|c|c|c|c|c|}
\hline & Date & Participants & Thème & Points abordés & $\begin{array}{l}\text { Questionnements, besoins identifiés } \\
\text { et difficultés }\end{array}$ \\
\hline EA1 & $16 / 11 / 2016$ & $\begin{array}{l}13 \text { participants: } \\
-6 \text { chercheurs } \\
-4 \text { structures d'appui } \\
-3 \text { institutions de l'État } \\
\text { décentralisées et déconcentrées }\end{array}$ & L'école-acteurs & $\begin{array}{l}\text { - Définition du concept } \\
\text { d'école-acteurs et présentation } \\
\text { de ses rôles et objectifs } \\
\text { - Thèmes à aborder } \\
\text { - Organisation: identifier les } \\
\text { personnes à mobiliser } \\
\text { - Convenir de l'enjeu à traiter } \\
\text { (la pollution diffuse) } \\
\text { et validation }\end{array}$ & $\begin{array}{l}\text { - L'approche territoriale/approche } \\
\text { filière. } \\
\text { - Comment s'assurer d'une bonne } \\
\text { représentation des acteurs au sein } \\
\text { des EA? }\end{array}$ \\
\hline EA2 & 09/03/2017 & $\begin{array}{l}28 \text { participants : } \\
-14 \text { chercheurs } \\
-9 \text { structures d'appui } \\
-4 \text { institutions de l'État } \\
\text { décentralisées et déconcentrées } \\
-1 \text { producteur et transformateur }\end{array}$ & $\begin{array}{l}\text { Les innovations } \\
\text { techniques }\end{array}$ & $\begin{array}{l}\text { - Caractérisation de } \\
\text { l'utilisation des pesticides } \\
\text { - Les innovations disponibles } \\
\text { pour une diminution: } \\
\text { paillage, post récolte, sarclage } \\
\text { mécanique et } \\
\text { accompagnement à la } \\
\text { transition agroécologique } \\
\text { - Dispositifs mis en œuvre }\end{array}$ & $\begin{array}{l}\text { - Comment évaluer les impacts? et } \\
\text { surtout sur le long terme? } \\
\text {-Quels freins au développement des } \\
\text { innovations? } \\
\text { - Comment les innovations } \\
\text { proposées par les institutions sont- } \\
\text { elles diffusées et comment sont- } \\
\text { elles perçues par les agriculteurs? } \\
\text { - Intérêt et importance de coupler } \\
\text { l'EA et les dispositifs } \\
\text { d'expérimentation. }\end{array}$ \\
\hline EA3 & $15 / 06 / 2017$ & $\begin{array}{l}22 \text { participants : } \\
-12 \text { chercheurs } \\
-4 \text { structures d'appui } \\
-3 \text { institutions de l'État } \\
\text { décentralisées et déconcentrées } \\
-1 \text { producteur et transformateur } \\
-2 \text { particuliers et associations de la } \\
\text { société civile }\end{array}$ & $\begin{array}{l}\text { Sensibiliser sans } \\
\text { culpabiliser }\end{array}$ & $\begin{array}{l}\text { - Préciser les règles du jeu de } \\
\text { l'EA, (écoute, respect d'autrui } \\
\text { et reconnaissance des } \\
\text { positions divergentes) } \\
\text { - Informer sans inquiéter ni } \\
\text { culpabiliser } \\
\text { - Accompagner au } \\
\text { changement de pratiques }\end{array}$ & $\begin{array}{l}\text { - Difficultés à mobiliser. } \\
\text { - Transformation de l'information. } \\
\text { - Confrontation des visions à long } \\
\text { terme (projet de recherche et } \\
\text { développement) et des visions à } \\
\text { court terme (inquiétudes de la } \\
\text { population). }\end{array}$ \\
\hline$\overline{\text { EA5 }}$ & $15 / 02 / 2019$ & $\begin{array}{l}34 \text { participants : } \\
-14 \text { chercheurs } \\
-5 \text { structures d'appui } \\
-6 \text { institutions de l'État } \\
\text { décentralisées et déconcentrées } \\
-3 \text { producteurs et transformateurs } \\
-6 \text { particuliers et associations de la } \\
\text { société civile }\end{array}$ & $\begin{array}{l}\text { L'agriculture } \\
\text { guadeloupéenne } \\
\text { et les attentes de } \\
\text { la population }\end{array}$ & $\begin{array}{l}\text { - Les attentes de la population } \\
\text { guadeloupéenne vis-à-vis de } \\
\text { l'agriculture locale } \\
\text { - Les réponses de cette } \\
\text { agriculture (évolutions des } \\
\text { systèmes de production selon } \\
\text { des normes, le marché et les } \\
\text { attentes des consommateurs) }\end{array}$ & $\begin{array}{l}\text { - Comment redonner confiance dans } \\
\text { les produits agricoles } \\
\text { guadeloupéens? } \\
\text { - Les importations sont-elles un } \\
\text { frein au développement de la } \\
\text { diversification? } \\
\text { - Nécessité de développer une } \\
\text { communication positive du métier } \\
\text { d'agriculteur. } \\
\text { - Les collectivités locales n'appuient } \\
\text { pas le développement de } \\
\text { l'agriculture guadeloupéenne. }\end{array}$ \\
\hline
\end{tabular}


Tableau 1. (suite).

\begin{tabular}{|c|c|c|c|c|c|}
\hline & Date & Participants & Thème & Points abordés & $\begin{array}{l}\text { Questionnements, besoins identifiés } \\
\text { et difficultés }\end{array}$ \\
\hline EA6 & 20/09/2019 & $\begin{array}{l}43 \text { participants : } \\
-15 \text { chercheurs } \\
-6 \text { structures d'appui } \\
-11 \text { institutions de l'État } \\
\text { décentralisées et déconcentrées } \\
-6 \text { producteurs et transformateurs } \\
-5 \text { particuliers et associations de la } \\
\text { société civile }\end{array}$ & $\begin{array}{l}\text { Quelles } \\
\text { perspectives pour } \\
\text { l'agriculture } \\
\text { guadeloupéenne? }\end{array}$ & $\begin{array}{l}\text { - La diversité des difficultés } \\
\text { rencontrées aujourd'hui par } \\
\text { l'agriculture guadeloupéenne } \\
\text { - Les leviers des filières les } \\
\text { mieux structurées (focus sur } \\
\text { les filières canne et banane) } \\
\text { - Les expériences alternatives }\end{array}$ & $\begin{array}{l}\text { - Nécessité de penser ensemble, et } \\
\text { sur le long terme pour construire } \\
\text { l'agriculture de demain. } \\
\text { - Nécessité de définir la } \\
\text { «diversification» et de reconnaître } \\
\text { la diversité de situations derrière ce } \\
\text { mot. } \\
\text { - Développer l'accompagnement de } \\
\text { cette diversité de producteurs. } \\
\text { - Comprendre les aides et les } \\
\text { niveaux de décision. }\end{array}$ \\
\hline EA7 & $15 / 11 / 2019$ & $\begin{array}{l}28 \text { participants : } \\
-7 \text { chercheurs } \\
-6 \text { structures d'appui } \\
-5 \text { institutions de l'État } \\
\text { décentralisées et déconcentrées } \\
-7 \text { producteurs et transformateurs } \\
-3 \text { particuliers et associations de la } \\
\text { société civile }\end{array}$ & $\begin{array}{l}\text { Le système } \\
\text { alimentaire } \\
\text { guadeloupéen }\end{array}$ & $\begin{array}{l}\text { - Le projet DIAG'Alim: } \\
\text { enjeux du système alimentaire } \\
\text { guadeloupéen } \\
\text { - La coordination des actions } \\
\text { au niveau du territoire } \\
\text { - Exemples d'initiatives } \\
\text { locales en agro- } \\
\text { transformation, restauration, } \\
\text { promotion de produits locaux, } \\
\text { lutte contre le gaspillage } \\
\text { alimentaire, etc. }\end{array}$ & $\begin{array}{l}\text { - Comment améliorer la traçabilité } \\
\text { - Informer et accompagner les } \\
\text { porteurs de projet. } \\
\text { - Faciliter l'approvisionnement en } \\
\text { produits locaux. } \\
\text { - Éduquer les consommateurs. } \\
\text { - Développer les canaux de } \\
\text { distribution. } \\
\text { - Besoin d'un outil financier } \\
\text { facilitant l'accès aux aides (avance } \\
\text { de trésorerie) et les transitions. }\end{array}$ \\
\hline
\end{tabular}

acceptant la responsabilité sociétale collective de l'utilisation des pesticides. Les évolutions des systèmes agricoles pour mieux répondre «aux attentes des populations en relation à l'agriculture», ont ensuite été abordées en analysant les fonctions multiples de l'agriculture, ce qui a remis partiellement en cause l'organisation par filières. La réflexion devient globale en traitant des "perspectives pour l'agriculture guadeloupéenne» dans une approche guidée par l'élaboration de «systèmes d'alimentation».

Les propositions ont été faites par les participants dans les débats. Elles ont été élaborées collectivement, même si elles n'ont jamais fait l'objet de décisions formelles. L'équipe d'animation avait prévu de rassembler les synthèses dans un document qui devait être validé par une école-acteurs en 2020, avant diffusion. Les propositions peuvent être regroupées autour de trois grands objectifs.

Un premier objectif est de mettre au point des itinéraires techniques et de garantir leur maîtrise par les agriculteurs. Ce travail a commencé mais il doit se renforcer : i) en prenant en compte les situations spécifiques pour analyser les possibilités d'innovation; ii) en s'appuyant sur les expériences et initiatives existantes, tant techniques, en les caractérisant et en les référençant, qu'institutionnelles (réseaux Delphy, Rita, associations, groupements...).

Un deuxième objectif, moins consensuel, concerne la reconfiguration de l'appui technique au sens large. L'objectif d'une meilleure coopération entre institutions pour mieux organiser les appuis sur l'ensemble du territoire est accepté par tous. Reste à définir les contenus et les modalités.

Un troisième objectif est de poursuivre le débat autour des fonctions de l'agriculture en Guadeloupe, en analysant le paradoxe entre une forte demande d'autonomie alimentaire et une agriculture principalement vouée à l'exportation. Ce dernier point est polémique, les divergences sont grandes, donc nécessitent plus de débat.

\section{Retour d'expériences et perspectives}

\subsection{Un processus d'évaluation incomplet}

Une évaluation a été réalisée courant 2019 en mobilisant trois outils: l'analyse des ressentis exprimés lors des évaluations en fin de chaque session; un questionnaire envoyé à l'ensemble des personnes qui ont participé au moins à une session de l'EA; 19 entretiens (dont 6 auprès d'agriculteurs) sur la pollution diffuse, qui traitaient aussi d'autres sujets que l'EA. 4 personnes ont répondu à la fois au questionnaire et à l'entretien. Seules 17 réponses au questionnaire ont pu être traitées, empêchant une réelle analyse statistique. Néanmoins, quelques tendances peuvent être identifiées. Pour une très grande majorité des répondants (plus de 9 sur 10), l'EA est un lieu de rencontres et d'échanges original, sans contraintes, où la parole est libre, permettant de travailler de manière différente. Mais 3 répondants sur 4 considèrent que les participants manquent d'informations pour débattre. Ces données ont contribué à l'auto-évaluation que l'équipe d'animation a menée.

\subsection{Constitution d'une communauté de médiateurs?}

L'intérêt pour l'EA s'est affirmé au fur et à mesure des sessions. Le chiffre de la participation (13 à 43 participants) a 
évolué positivement (sur un nombre d'invitations passé de 35 à 138). Néanmoins, le turn-over a été important, même si un petit noyau d'une quinzaine de personnes était toujours présent. Les participants adhèrent à l'idée, acceptent les formes d'animation et reconnaissent l'intérêt qu'ils ont à aborder, par le débat, la question de la pollution diffuse.

L'augmentation du nombre de participants est un indice d'un succès grandissant, même si la participation dépend du thème. L'EA voulait décloisonner les publics. L'objectif n'a été que partiellement atteint: les chercheurs et les représentants d'institutions de l'État sont plus nombreux que les agriculteurs.

\subsection{La qualité des débats}

Les questionnaires donnent quelques éléments sur la qualité du débat. Les agriculteurs se disent toujours intimidés : ils ont du mal à prendre la parole, se sentant illégitimes. Le renforcement des compétences n'a pas été à la hauteur des enjeux. Des efforts restent à faire sur la simplicité des langages dans un cercle majoritairement constitué «d'intellectuels». Si certains profitent des moments d'échanges pour exprimer leur point de vue personnel, d'autres se protègent toujours derrière des idées consensuelles ou des positionnements institutionnels. Un intervenant a ainsi préparé une présentation relativement critique sur les aides publiques, mais a demandé à un membre de l'équipe d'animation de la présenter.

\subsection{La question de la production de connaissances}

Beaucoup d'informations ont été transmises. Les participants reconnaissent qu'ils ont appris des exposés introductifs et de contextualisation, des études de cas et des témoignages. Mais les gains en connaissances, individuels et collectifs, sont encore à mesurer. Cette évaluation demandera la mise en place de dispositifs fins (Argyris et Schön, 1996).

\subsection{La question de l'impact}

Une critique concerne «l'après ». À quoi sert l'EA? Que faire de toutes les idées exprimées? Le manque de continuité entre les différentes sessions semble être un frein à la valorisation, et d'une certaine manière, à la «fidélisation » des acteurs.

De fait, à ce jour, les alliances attendues ne se sont encore pas constituées. Les analyses de la situation et les visions de l'avenir de l'agriculture restent divergentes (place des filières d'exportation; intérêt d'une agriculture de proximité ; agroécologie...). Ces divergences ont été constatées, parfois comprises, mais elles sont loin d'être aplanies.

\subsection{Perspectives}

Des propositions veulent renforcer les impacts. Certains acteurs souhaitent transformer les EA en une structure plus opérationnelle, assumant d'autres tâches: accompagnement des expériences dans les réseaux; communication et formation; coordination des activités; propositions de politiques publiques.
Ces évolutions ne pourront se mettre en œuvre que si l'EA se consolide en organisant mieux la production de connaissances. La production de synthèses, de fiches techniques, de témoignages, de vidéos est nécessaire pour capitaliser les expériences et les résultats des différentes sessions.

La principale difficulté est la pérennisation d'un tel dispositif. L'EA a été financée sur projet. Sa continuité en l'état n'était pas garantie au-delà de 2020, avant même la crise sanitaire. Alors que dire d'actions plus ambitieuses? La recherche des conditions de cette pérennisation (financement, institution gestionnaire...) serait un autre axe de travail.

\section{Conclusion}

En s'appuyant sur les principes de l'éducation populaire, l'école-acteurs cherche à accompagner un collectif dans la codéfinition de voies de développement agricole plus soutenable, en s'appuyant sur des partages d'expériences et une amélioration de la communication. L'exercice collectif de réflexivité est central. Le processus n'est qu'à son début. Il a manqué de moyens. S'il a retenu l'attention des participants, il mérite encore des améliorations, dans un temps long, celui de l'éducation et de l'apprentissage. En particulier, le renforcement des acteurs en asymétrie de pouvoir doit être réalisé de manière spécifique, intégrant ainsi approches critique et dialogique. L'expérience est récente et les enseignements restent parcellaires et mitigés. Malheureusement, l'expérience ne se poursuivra pas car la fragilité institutionnelle et la difficulté de travailler dans le temps long obèrent aujourd'hui de telles initiatives.

Remerciements. Le projet RIVAGE est un projet financé par des fonds européens Feder et la Région Guadeloupe, en partenariat avec le Bureau de recherches géologiques et minières (BRGM), le Centre de coopération internationale en recherche agronomique pour le développement (CIRAD), l'Institut national de recherche pour l'agriculture, l'alimentation et l'environnement (INRAE) et l'Université des Antilles.

\section{Références}

Argyris C, Schön D. 1996. Organizational learning II: Theory, method and practice. Reading, MA: Addison-Wesley, XXIX: 305 p. DOI: 10.1177/103841119803600112.

Barbier R, Larrue C. 2011. Démocratie environnementale et territoires : un bilan d'étape. Participations 1(1): 67-104. DOI: 10.3917/parti.001.0067.

Barbier R, Roussary A, eds. 2016. Les territoires de l'eau potable : chronique d'une transformation silencieuse (1970-2015). Versailles : Quae, $141 \mathrm{p}$.

Barnaud C, d'Aquino P, Daré W, Mathevet R. 2016. Dispositifs participatifs et asymétries de pouvoir : expliciter et interroger les positionnements. Participations 16(3): 137-166. DOI: 10.3917/ parti.016.0137.

Bonin M, Cathelin C. 2014. Conversion environnementale de la production bananière guadeloupéenne : une stratégie politique et économique. Économie rurale 341(3): 63-82. DOI: 10.4000/ economierurale.4329.

Cooke W, Kothari U, eds. 2001. Participation: the new tyranny? London: Zed Books, 62 p. 
DAAF Guadeloupe. 2018a. La culture de la canne à sucre. Résultats de l'enquête statistique réalisée en 2014 auprès de 185 planteurs en Guadeloupe. Agreste Guadeloupe 8: 4.

DAAF Guadeloupe. 2018b. La culture de banane. Premiers résultats de l'enquête statistique réalisée en 2016 auprès de 128 bananeraies en Guadeloupe. Agreste Guadeloupe 9: 4.

Deffontaines L, Mottes C, Della Rossa P, Lesueur-Jannoyer M, Cattan P, Le Bail M. 2020. How farmers learn to change their weed management practices: Simple changes lead to system redesign in the French West Indies. Agricultural Systems 179: 102769. DOI: 10.1016/j.agsy.2019.102769.

Della Rossa P, Le Bail M, Mottes C, Lesueur-Jannoyer M, Cattan P. 2020. Innovations developed within supply chains hinder territorial ecological transition: the case of a watershed in Martinique. Agronomy for Sustainable Development 40(10): 16. DOI: 10.1016/ j.agsy.2019.102769.

Euvé F. 2018. Une pédagogie humaniste. Études 1: 4-6.

Faysse N. 2006. Troubles on the way: An analysis of the challenges faced by multi-stakeholder platforms. Natural Resources Forum 30: 219-229. DOI: 10.1111/j.1477-8947.2006.00112.x.

Fouilleux È. 2000. Entre production et institutionnalisation des idées. La réforme de la Politique agricole commune. Revue française de science politique 50(2): 277-306. DOI: 10.3406/rfsp.2000.395468.

Gorry A. 2012. Les postures des animateurs et animatrices scientifiques quant au dialogue «sciences en/et société». Mémoire de l'Université de Montréal, Québec.

Jobert B (dir.). 1994. Le tournant néo-libéral en Europe. Paris: L'Harmattan, $328 \mathrm{p}$.

Leclerc G. 2000. Lire l'agir pédagogique : une lecture épistémologique. Revue des sciences de l'éducation 26(2): 243-262. DOI: 10.7202/000122ar.

Lesueur-Jannoyer M, Cattan P, Monti D, Saison C, Voltz M, Woignier T, et al. 2012. Chlordécone aux Antilles : évolution des systèmes de culture et leur incidence sur la dispersion de la pollution. Agronomie, Environnement et Sociétés 2(1): 45-58.

Lesueur-Jannoyer M, Cattan P, Woignier T, Clostre F. 2016. Crisis management of chronic pollution: contaminated soil and human health. Boca Raton: CRC Press, 290 p.

Mathevet R, Antona M, Barnaud C, Fourage C, Trébuil G, Aubert S. 2010. Contextes et dépendances des contextes d'accompagnement. In: Etienne M, ed. La modélisation d'accompagnement; une démarche participative en appui au développement durable. Versailles : Quae, pp. 103-123.
Maurel C. 2001. Éducation populaire et travail de la culture. Éléments d'une théorie de la praxis. Paris: L'Harmattan, $170 \mathrm{p}$.

Meiffren O, Oudin B, Gallois-Bride F, Galan MB. 2014. Evaluation du Plan Banane Durable 1. Livrable 3, Blezat consulting. [Online]. https://agriculture.gouv.fr/evaluation-du-plan-banane-durablebilan-de-la-mise-en-oeuvre.

Mongruel R, Prou J, Ballé-Béganton J, Lample M, Vanhoutte-Brunier A, Réthoret $\mathrm{H}$, et al. 2011. Modeling soft institutional change and the improvement of freshwater governance in the coastal zone. Ecology and Society 16(4): 15. DOI: 10.5751/ES-04294-160415.

Muller P. 2000. L'analyse cognitive des politiques publiques : vers une sociologie politique de l'action publique. Revue Française de Science Politique 50(2): 189-208. DOI: 10.3406/ rfsp.2000.395464.

Multigner L, Kadhel P, Rouget F, Blanchet P, Cordier S. 2016. Chlordecone exposure and adverse effects in French West Indies populations. Environmental Science and Pollution Research 23: 3 8. DOI: $10.1007 / \mathrm{s} 11356-015-4621-5$.

Olivier de Sardan JP, Bierschenk T. 1994. Les courtiers locaux du développement. Bulletin de l'APAD 5.

Revault d'Allonnes M. 2011. L'incertitude démocratique et la crise actuelle de la démocratie. Éthique publique [En ligne], 13(2). DOI: 10.4000/ethiquepublique.743.

Roling NG, Wagemakers A. 1998. A new practise: facilitating sustainable agriculture. In: Roling NG, Wagemakers A (dir.). Facilitating Sustainable Agriculture: Participatory learning and adaptive management in times of environmental uncertainty. Cambridge: Cambridge University Press, pp. 3-22.

Salles D, Barraqué B, Busca D, Garin P. 2006. Eau des villes, eau des champs. Négociation territoriale et génie de l'environnement. Rapport au programme CNRS PIDUD, CERTOP-UTM.

Tonneau JP, Piraux M, Coudel É. 2011. Quelles innovations territoriales dans des territoires marginalisés au Nordeste du Brésil ? Cahiers Agricultures 20(3): 235-240. DOI: 10.1684/ agr.2011.0487.

Ulrich W. 2003. Beyond methodology choice: Critical systems thinking as critically systemic discourse. Journal of the Operational Research Society 54: 325-342. DOI: 10.1057/palgrave. jors.2601518.

Werdenberg F, Arrault S. 2014. Les écoles-chercheurs, outil au service de l'exploration de fronts de sciences interdisciplinaires. Rochebrune : INRA. https://www.gemass.fr/dphan/rochebrune14/ propositions/rochebrune14-26.pdf.

Citation de l'article : Tonneau J-P, Bonnal V, Bourgoin J, Cheval A, Jannoyer M, Chéry J-P, Cattan P. 2021. Les écoles-acteurs en Guadeloupe, un espace de débat sur les pollutions agricoles et le devenir de l'agriculture. Cah. Agric. 30: 9. 